\title{
On the Extraction of the Valid Speech-Sound by the Merging Algorithm with the Discrete Wavelet Transform
}

\author{
Jin Ok Kim ${ }^{1}$, Han Wook Paek ${ }^{2}$, Chin Hyun Chung ${ }^{2}$, Jun Hwang ${ }^{3}$, and \\ Woongjae Lee $^{3}$
}

1 School of Information and Communication Engineering, Sungkyunkwan University, 300, Chunchun-dong, Jangan-gu, Suwon, Kyunggi-do, 440-746, KOREA

jinny@ece.skku.ac.kr

2 Department of Information and Control Engineering, Kwangwoon University, 447-1, Wolgye-dong, Nowon-gu, Seoul, 139-701, KOREA

chung@kw.ac.kr

3 Division of Information and Communication Engineering, Seoul Women's University, 126, Kongnung2-dong, Nowon-gu, Seoul, 139-774, KOREA wjlee@swu.ac.kr

\begin{abstract}
A valid speech-sound block can be classified to provide important information for speech recognition. The classification of the speechsound block comes from the MRA(multi-resolution analysis) property of the DWT(discrete wavelet transform), which is used to reduce the computational time for the pre-processing of speech recognition. The merging algorithm is proposed to extract valid speech-sounds in terms of position and frequency range. It needs some numerical methods for an adaptive DWT implementation and performs unvoiced/voiced classification and denoising. Since the merging algorithm can decide the processing parameters relating to voices only and is independent of system noises, it is useful for extracting valid speech-sounds. The merging algorithm has an adaptive feature for arbitrary system noises and an excellent denoising SNR (signal-to-noise ratio).
\end{abstract}

\section{Introduction}

In the case of building a speech recognition system, we spend a lot of time in tuning up the pre- or post-process of an imported speech because the quality of the pre- or post-process is sometimes dependent on the range of the speech that is coming in or out. Even a valid speech may overlap with some high frequency noises. The Fourier transform method to extract valid speech takes a lot of processing time and is limited in the frequency analysis. Since a zero-crossing method is very sensitive to external noises, it could be ineffective [1].

In order to obtain reliable and valid speech, the MRA property is proposed because it can track and reconstruct the unvoiced phonemes in speech. The merging algorithm is proposed to extract valid speech data, especially the unvoiced speech-sound blocks that consider position and frequency range. 
When extracting a valid speech-sound block, much work has to be devoted to the search of the frequency range included in the voiced/unvoiced speech and in each of its positions. However, the simultaneous analysis of the frequency and time (position) can hardly be obtained by the Fourier transform [2] [3]. Extracting data from the desired frequency range of the original signal by the DWT involves considering the denoising effect and the compression effect on the speech signal [4] [5] [6] [7]. Thus, the DWT is used for simultaneous analysis and for a decrease in its computational amount.

The merging algorithm is therefore proposed to discriminate between valid phonemes and silence.

\section{Discrete Wavelet Transform}

In general, a wavelet is a small wave which has its energy concentrated in time. It can be used to give a tool for the analysis of transient, nonstationary, or timevarying phenomena. A wavelet still maintains an oscillating wavelike characteristic but also has the ability to allow simultaneous time and frequency analysis with a flexible mathematical foundation [4] [8]. The two-dimensional parameters are achieved from a function called "the generating wavelet" or "mother wavelet" $[9], \psi(t)$ by

$$
\begin{array}{ll}
\psi_{j, k}(t)=2^{j / 2} \psi\left(2^{j} t-k\right), & j, k \in \mathbf{Z} \\
\varphi_{j, k}(t)=2^{j / 2} \varphi\left(2^{j} t-k\right), & j, k \in \mathbf{Z}
\end{array}
$$

where $\mathbf{Z}$ is the set of all integers and the factor $2^{j / 2}$ maintains a constant norm. The parameters of the time or space location by $k$ and the frequency or scale (actually the logarithm of scale) by $j$ turn out to be extraordinarily effective [10]. The goal is to generate a set of expansion functions so that any signal $L^{2}(R)$ can be represented by the series

$$
f(t)=\sum_{j, k} a_{j, k} 2^{j / 2} \psi\left(2^{j} t-k\right)
$$

where the two-dimensional set of coefficients $a_{j, k}$ is called the discrete wavelet transform of $f(t)$. The MRA property of the DWT is well defined in the implementation [11], which is formulated by requiring a nesting of the spanned spaces as

$$
\cdots \subset V_{-1} \subset V_{0} \subset V_{1} \subset V_{2} \subset \cdots \subset L^{2}
$$

or

$$
V_{j} \subset V_{j+1} \quad \text { for all } j \in \mathbf{Z}
$$

with

$$
\begin{gathered}
V_{-\infty}=\{0\}, \quad V_{\infty}=L^{2} \\
f(t)=\sum_{k} c_{J_{0}}(k) \varphi_{J_{0}, k}(t)+\sum_{k} \sum_{j=J_{0}}^{J-1} d_{j}(k) \psi_{j, k}(t)
\end{gathered}
$$




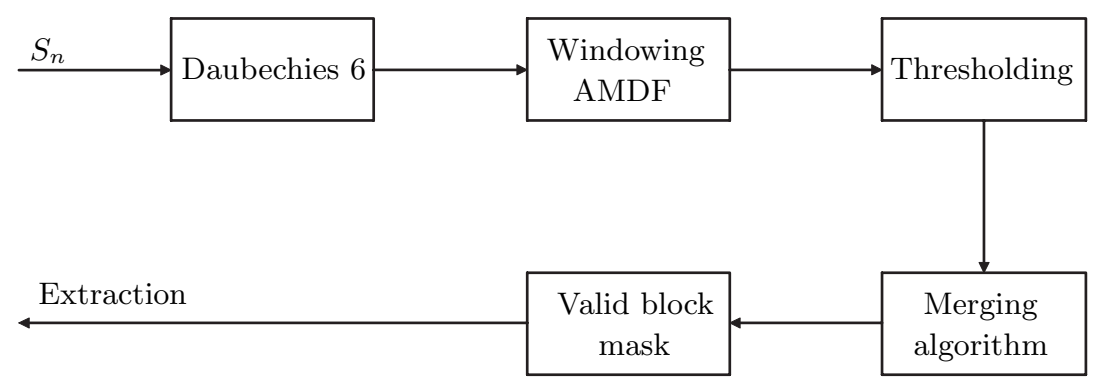

Fig. 1. Processing diagram

\section{Merging Algorithm}

A band in which the vowels or voiced sounds are dominant in the speech signal is selected for analysis. The statistical results of many vowels of adult males and females indicate that the first formant frequency does not exist below approximately $100 \mathrm{~Hz}$ [10] [12] [13]. However, the unvoiced sound is spread over all frequencies as noise. Thus, when searching for valid unvoiced speech sounds, one can make the following assumptions;

- The energy of noise is less than the valid voiced sound.

- The valid unvoiced sound wraps the voiced sound.

- The valid unvoiced sound spreads over the band that is less than about 3 $\mathrm{kHz}$.

In general, a speech sound obtained by a microphone includes less noise than a valid unvoiced speech sound [14] [15]. However, if a system is constructed in real fields, denoising high frequency noises is included [16]. The merging algorithm is proposed to focus on denoising and the extraction of the unvoiced speech-sound block.

The silence-discrimination method that uses energy and zero-crossing is useful in the case of an extremely high SNR (signal-to-noise ratio). However, such ideal conditions are not practical for most application environments in which a neighboring device occasionally generates high frequency noises. The merging algorithm is used in the extraction process with a position array of each phoneme and a higher frequency of unvoiced speech than of voiced speech. Several processes are dedicated to the extraction of the valid block in order to merge each phoneme block. These are processed in terms of the phoneme-block to increase the discrimination property.

Figure 1 shows a block diagram of the merging algorithm and its detailed algorithm is described in Algorithm 1.

Figure 2 shows an array of phonemes. The signal is merged with the information and the pre-defined rules. In general, a person produces speech at an average rate of about 10 phonemes per second. Therefore, for classification purposes, at 


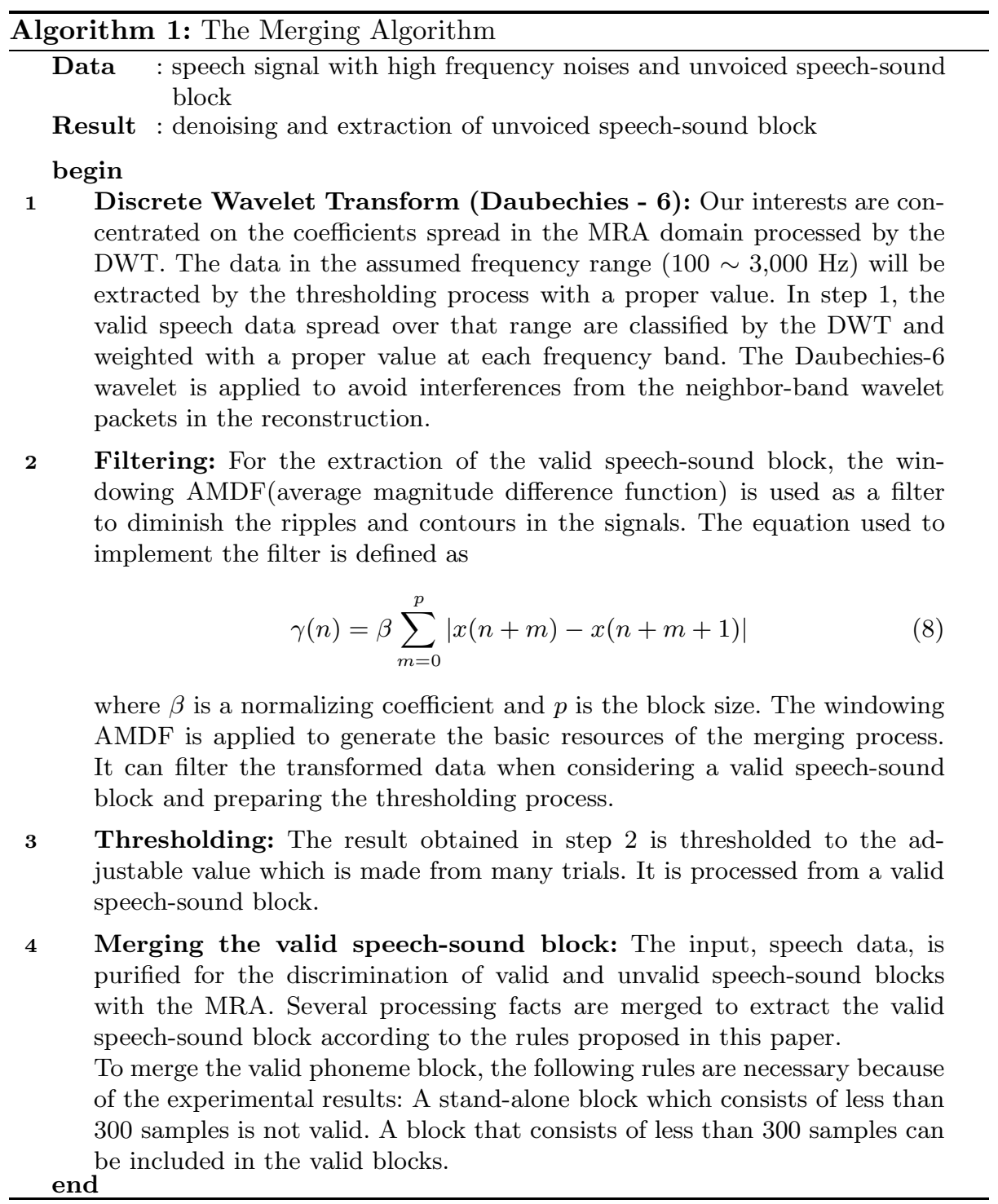

least 1,000 samples are needed in a sampling frequency of 11,025 Hz. Except for the detail classification, the minimum size of a valid block frame is suggested at $300 \sim 500$ samples. To determine the valid block, we should consider the energy and position of each frame simultaneously. Since the valid block is extracted by the merging rules described, its valid speech-sound block can be classified. 
Table 1. Denoising SNR.

\begin{tabular}{|c|c|}
\hline Original Signal & 0.962391 \\
\hline Denoised Signal $+6 \mathrm{kHz}$ Sin & 254.214 \\
\hline
\end{tabular}

\section{Experiment Results}

The merging algorithm is implemented to get the sample data through a microphone within the sampling rate of $11,025 \mathrm{~Hz}$.

To describe the DWT's extraction performance of the desired frequency range, Fig. 3 shows the denoising(filtering a band range's data) effect of the DWT.

$$
\mathbf{S N R}=\frac{E\left[x^{2}(n)\right]}{E\left[e^{2}(n)\right]}=\frac{\sum_{n} x^{2}(n)}{\sum_{n} e^{2}(n)}
$$

Table 1 shows that the noises have no interference following the extraction of the desired band, if the desired frequency range is added.

Figure 4 shows that the merging algorithm has an adaptive feature for arbitrary system noises. The original signal includes the high frequency system noises, whereas in the result signal, the merging algorithm shows an improved extraction performance, especially when denoising the higher frequency noise.

Figures 5 and 6 show that the original signals compounded with $80 \mathrm{~Hz}$ and $6,000 \mathrm{~Hz}$ sin wave are processed by the merging algorithm. They also show that the merging algorithm is not disturbed by an unexpected system interference.

Table 2 shows a comparison of the merging algorithm with the "Zero-Crossing \& Energy Consideration". The merging algorithm is independent of system noises and it has an adaptive feature for spot noises.

\section{Conclusion}

In general, high frequency noises included in a normal speech stream are difficult to remove from the speech stream. Because an unvoiced phoneme seems like a

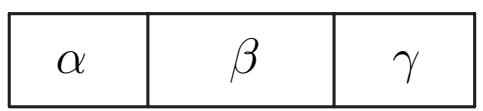

$\alpha$ : unvoiced speech

$\beta$ : voiced speech

$\gamma$ : silence range

Fig. 2. The position array of each phoneme 


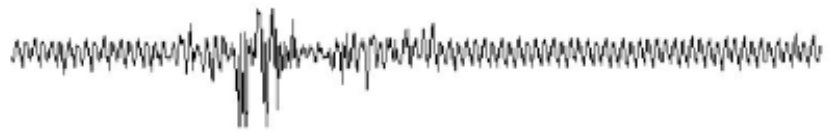

(a) a speech signal of "six" added with system noises

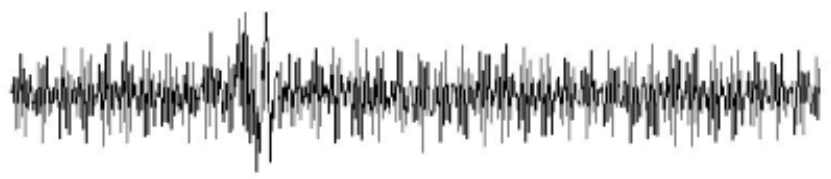

(b) a speech signal of "six" added with system noises and $6 \mathrm{kHz}$ sine wave

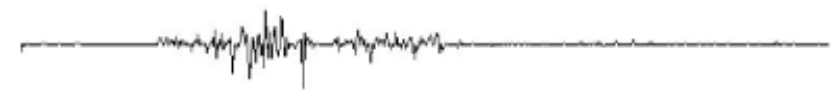

(c) denoising the speech signal of "six" added with system noises

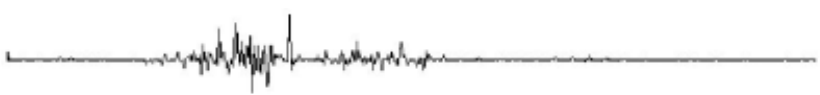

(d) denoising the speech signal of "six" added with system noises and $6 \mathrm{kHz}$ sine wave

Fig. 3. Denoising.

Table 2. Property comparison.

\begin{tabular}{|c|c|c|}
\hline Item & $\begin{array}{c}\text { Zero-crossing \& } \\
\text { energy consideration }\end{array}$ & Merging algorithm \\
\hline System dependency & Higher & Lower \\
\hline Spot-noise & Non-Adaptive & Adaptive \\
\hline Signal analysis & None & DWT's MRA \\
\hline Unvoiced phoneme & $\begin{array}{c}\text { Dependent on } \\
\text { System }\end{array}$ & $\begin{array}{c}\text { Dependent on } \\
\text { the frequency range }\end{array}$ \\
\hline
\end{tabular}


high frequency noise, it may be removed during denoising. A low frequency noise (hum noise), on the other hand, may come from a circuitry imbalance, a wrongly designed ground point in $\mathrm{PCB}$, or imbalance among the parts mounted on a board. This experiment results show that the merging algorithm is very robust against external effects. Since the merging algorithm proposed in this paper is based on the MRA with the DWT, its computation seems complicated. However, because the basic computation of the DWT is processed by convolution, it can be done more quickly by the pipeline processing of convolution. Since the other methods must decide the processing parameters of system noises and voices, they can hardly tune themselves. The merging algorithm is useful for extracting valid speech-sounds since it can decide the processing parameters relating to voices only and is independent of system noises. The merging algorithm has an adaptive feature for arbitrary system noises and an excellent denoising SNR.

\section{References}

1. Goldberg, R., Riek, L.: A Practical Handbook of Speech Coders. CRC Press, Boca Raton, FL (2000)

2. Goswami, J.C., Chan, A.K.: Fundamentals of Wavelets: Theory, Algorithms and Applications. John Wiley \& Sons, New York (1999)

3. Teolis, A.: Computational Signal Processing with Wavelets. Springer Verlag, New York (1998)

4. Burrus, C.S., Gopinath, R.A., Guo, H.: Introduction to Wavelets and Wavelet Transforms: A Primer. Prentice Hall, New Jersey (1997)

5. Marzetta, T.L.: A new interpretation for capon's maximum likelihood method of frequency-wavenumber spectral estimation. IEEE Trans. Acoustics, Speech, and Signal Processing 31 (1983)

6. Deller, J.R., Hansen, J.H.L., Proakis, J.G.: Discrete-Time Processing of Speech Signals. IEEE Press, New York (2000)

7. Donoho, D.L.: Denoising by soft-thresholding. IEEE Trans. Information Theory 41 (1995)

8. Abbate, A., Decusatis, C.M., Das, P.K.: Wavelets and Subband: Fundamentals and Applications. Birkhauser, Stuttgart, Germany (2001)

9. Ogden, R.T.: Essential Wavelets for Statistical Applications and Data Analysis. Springer Verlag, New York (1996)

10. Parsons, T.W.: Voice and Speech Processing. McGraw-Hill, New York (1986)

11. Furui, S.: Digital Speech Processing, Synthesis and Recognition. 2nd edn. Marcel Dekker, New York (2001)

12. Jurasfky, D., Martin, J.H., Linden, K.V., Jurafsky, D.: Speech and Language Processing: An Introduction to Natural Language Processing, Computational Linguistics and Speech Recognition. Prentice Hall, New Jersey (2000)

13. Morgan, N., Gold, B.: Speech and Audio Signal Processing: Processing and Perception of Speech and Music. John Wiley \& Sons, New York (1999)

14. Rabiner, L., Juang, B.H., Juang, B.H.: Fundamentals of Speech Recognition. Prentice Hall, New Jersey (1993)

15. Huang, X., Acero, A., Hon, H.W., Reddy, R.: Spoken Language Processing. Prentice Hall, New Jersey (2001)

16. Quatieri, T.F.: Discrete-Time Speech Signal Processing. Prentice Hall, New Jersey (2001) 


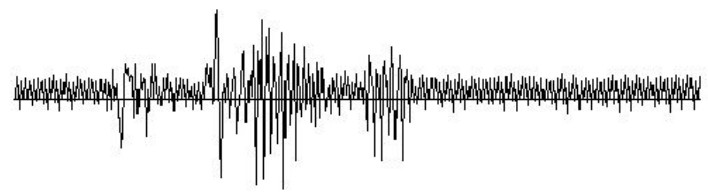

(a) a speech signal

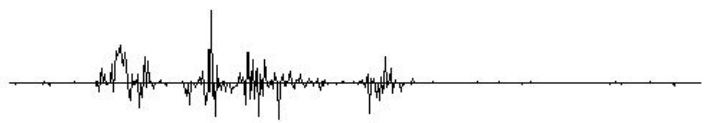

(b) a signal filtered by the DWT

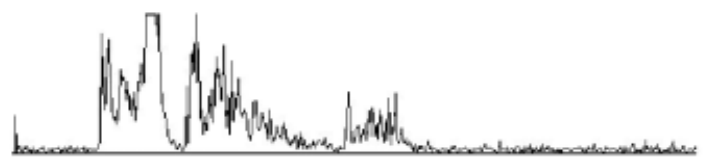

(c) a windowing AMDF

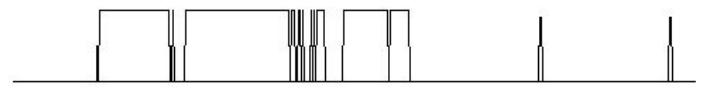

(d) a thresholding data

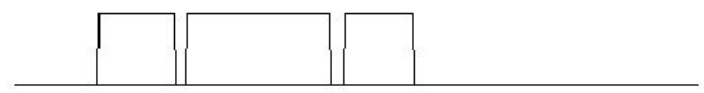

(e) an extraction mask

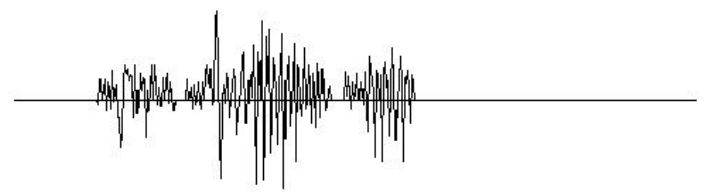

(f) an extracted signal

Fig. 4. Extraction. 


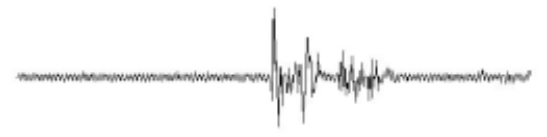

(a) a speech signal

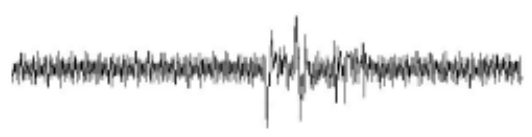

(b) a speech signal added with a noise of $80 \mathrm{~Hz}$

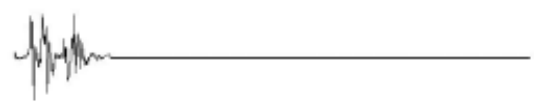

(c) a speech signal classified and compressed by the merging algorithm

Fig. 5. Classification and compression from the signal added with a noise of $80 \mathrm{~Hz}$

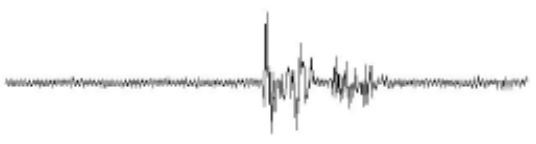

(a) a speech signal

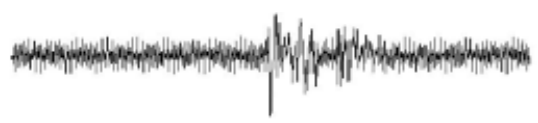

(b) a speech signal added with a noise of $6 \mathrm{kHz}$

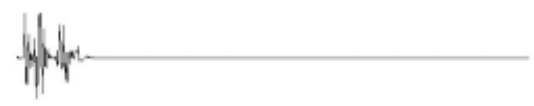

(c) a speech signal classified and compressed by the merging algorithm

Fig. 6. Classification and compression from the signal added with a noise of $6 \mathrm{kHz}$ 